\title{
Deep Learning Innovations in Recommender Systems
}

\author{
Bilal Ahmed \\ College of Information and \\ Computer
}

Taiyuan University of Technology

\author{
Waqar Hussain \\ College of Information and \\ Computer
}

Taiyuan University of Technology

\author{
Li Wang \\ College of Information and \\ Computer \\ Taiyuan University of Technology
}

\author{
Muhammad Amjad \\ College of Information and \\ Computer
}

Taiyuan University of Technology

\author{
Syed Badar-ud-Duja \\ College of Information and \\ Computer \\ Taiyuan University of \\ Technology
}

\author{
M. Abdul Qadoos Bilal \\ College of Information and \\ Computer \\ Taiyuan University of \\ Technology
}

\begin{abstract}
Recommender systems are one of the best choices to cope with the problem of information overload. These systems are commonly used in recent years help to match users with different items. As more data is available on the internet traditional methods suffer from challenges like accuracy and scalability. Deep learning a state of art machine learning method also achieve promising performance in the field of recommender system. In this study we provide an overview of traditional approaches their limitations and then discuss about the aspects of deep learning used in the recommender system domain to improve the accuracy in recommender system domains. These deep recommender systems can be used to understand the demands of users and improve the value in recommendations.
\end{abstract}

\section{General Terms}

Recommendations Systems, Deep Learning, Recommendations Algorithms.

\section{Keywords}

Recommender Systems, Deep Learning, Neural Networks

\section{INTRODUCTION}

To overcome the problem of information overload the community used recommender systems in recent years. The amount of data available on the internet is increased exponentially. In the era of E- commerce the choices on the number of goods and facilities provided by different companies has to be increased intensely [1]. Now a days many people use recommender system in their daily life to deal with the problem of information overload. These systems produce recommendations for the relevant goods to help their users in many assessment making procedures [2]. With these systems consumers achieve the suitable product and facilities such as music, movies, books, foods, hospitals, restaurants and also different places for their visits [3]. Facebook has developed a recommender system to recommend people to tag friends in pictures. Several recommender system algorithms have been proposed so far to provide an affordable, personal and effective recommendation to meet the requirement of different customers. Recommender systems have becoming commonly used in recent years. A selection of different traditional methods has been offered to make recommendations like Collaborative Filtering, Content Based Filtering and Hybrid Filtering as shown in the figure 1 [4]. The article is organized as follows. Section 1 affords a brief background of traditional recommendation methods. Section 2 and 3 discusses about different architecture of recommender system with respect of deep learning. In section 4 we present a brief overview of strength of deep learning usage in recommender system. Section 5 provides concluding remarks and some guidelines for the upcoming research.

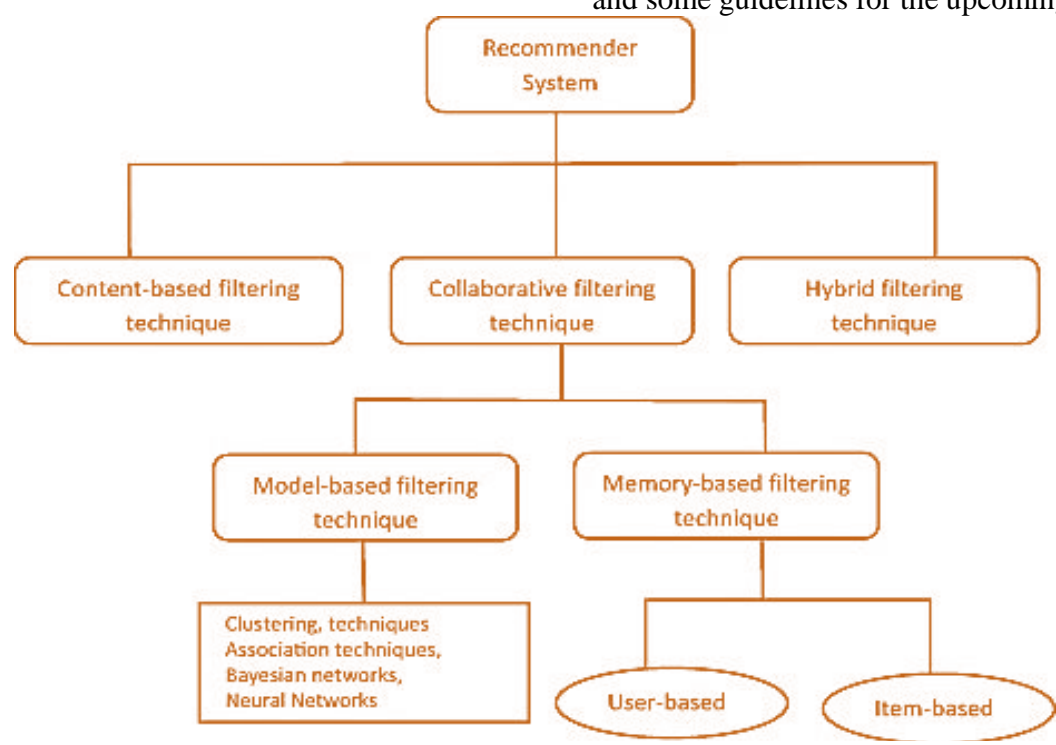

Fig 1: Different Methods for Recommender System 


\subsection{Collaborative Filtering}

It is the best successful approach in recommender systems that those peoples who are same on their tastes in the previous would also same in future. It contains some $\mathrm{m}$ users as $\mathrm{U}=$ $\{\mathrm{U} 1, \mathrm{U} 2, \mathrm{U} 3 \ldots \mathrm{Um}\}$ and some $\mathrm{n}$ type items $\mathrm{P}=\{\mathrm{P} 1$,

$\mathrm{P} 2, \mathrm{P} 3 \ldots . \mathrm{Pn}\}$. Then the method construct an $\mathrm{m} \times \mathrm{n}$ users and items matrix which contain the users ratings for that specific items also every entry $\mathrm{Ri}, \mathrm{j}$ is denoted by the rating given from user $\mathrm{Ui}$ for that specific items $\mathrm{Pj}$ as shown in the figure 2.

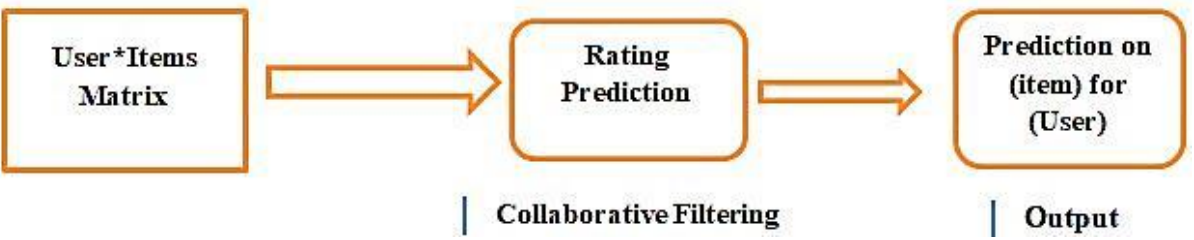

Fig 2: Working of Collaborative Filtering

These collaborative filtering models have two main approaches for the recommendation generation problem.

\subsubsection{Memory Based Methods}

Memory based methods are user based and item based. In memory based models recommendations are made based on the similarity values. Ratings are used to compute the similarity between users and items. To compute the similarity among items or users Pearson Correlation and Vector Cosine based similarity is used [5].

For example to calculate Pearson correlation between two user $\mathrm{u}$ and $\mathrm{v}$ is as follows.

$$
V_{i, j}=\frac{\sum_{u \in U}\left(r_{u, j}-r_{i}\right)\left(r_{u, j}-r_{j}\right)}{\sqrt{\sum_{u \in U}\left(r_{u, j}-\bar{r}_{l}\right)^{2}} \sqrt{\sum_{u \in U}\left(r_{u, j}-\bar{r}_{j}\right)^{2}}}
$$

Vector based cosine similarity computation between two things I and $\mathrm{j}$ can be derived as

$$
W_{i, j}=\cos (\vec{\imath}, \vec{\jmath})=\frac{\vec{\imath} \cdot \vec{\jmath}}{\|\vec{\imath}\| *\|\vec{\jmath}\|}
$$

\subsubsection{Model-Based Methods}

These methods build an offline method by applying data mining and machine learning techniques like decision trees, clustering, and singular value decomposition model [6].

\subsection{Content based Filtering}

These algorithms produced recommendations that are based upon the item features and profile of users. In this type of filtering method the main idea is to recommend things that a user is liked in the past. This approach is very efficient when you recommend an item that is newly inserted in the system. Although these systems are very efficient to recommending new items but they can't provide the personalize recommendation due to not enough data about the profile of user [7].

\subsection{Hybrid Recommender Systems}

Collaborative filtering methods and content based filtering methods both have some strengths and flaws. These hybrid systems combine both methods to overcome the restrictions of each method. A typical scenario is to apply content based statistics of a new item without the user rate in the collaborative filtering recommender systems [5]. Although these existing techniques are effective in producing recommendations but they quiet suffer from challenges like scalability, accuracy, and cold start problems. Deep learning has expressively affected the research way of recommender system in many aspects [8].

\section{DEEP LEARNING}

Deep learning is a technique used in the area of machine learning and is founded on several layers of representation by artificial neurons and it's also the new area of data mining research. Some main features that support deep learning as the state of art machine learning technique are big data and computational power. These models learns better representation when large amount of data is provided also graphical processing units are used to process the complex computation in deep learning models respectively [9].

\section{USE OF DEEP LEARNING IN RECOMMENDER SYSTEMS}

Different deep learning architecture for recommendations is as follows.

\subsection{Restricted Boltzmann machines for recommendation}

These machines has two types of layer one is visible layer also called softmax layer the other layer is called hidden layer. Restricted Boltzmann machines are using to extract features of users or product ratings in a recommender system. These are also used to model the correlation between user rating products and correlation between a user who rate a specific product to improve the accuracy in recommendations [10].

\subsection{Deep Belief Networks for Recommendation}

In content based recommendation methods deep belief networks are used to extract the unknown and useful features of music content in music recommendation system. These networks also used in text data to analyze the user preferences and classification tasks [11].

\subsection{Auto Encoders for Recommendation}

These networks compressed the given files with its encoder portion and reconstruct that data with its decoder part. These models are used in recommendation systems to acquire the non- linear image of user and item matrix and then rebuild it by defining the missing information. These methods are also used for dimensionality reduction and the extraction of latent features. To handle the problem of sparsity and scalability auto-encoders are specially used in the recommender system [12].

\subsection{Recurrent Neural Networks (RNN)}

Recurrent neural networks are used to process the sequence of information. These networks are utilized to integrate the current view webpage history and view order to provide more accurate recommendations. RNN are very effective on the recommendation coverage [13]. 


\subsection{Convolutional Neural Networks (CNN)}

Convolutional neural networks can also be used for different types of tasks like object classification and image recognition. CNNs are used in recommender systems to extract the visual features in any image to provide better recommendations [14].

\section{STRENGTHS OF DEEP LEARNING IN RECOMMENDATION MODELS}

\subsection{Nonlinearity}

Deep neural network models are nonlinear models while traditional models like matrix factorization models are linear models.in deep learning nonlinear activation functions like relu, tanh and sigmoid are used to model the non- linearity in data. Due to these functions it is possible to capture the complex patterns in data [15].

\subsection{Learning Representations}

These neural networks are used in learning the explaining factors and also some representations. With the help of these learning networks we can easily capture representation and explaining factors from input data. In recommender systems domain a huge amount of information about users and items are available on the internet. With the help of this huge information it is very easy to understand the users and items. With the help of deep learning it is very easy to learn all the features from the raw data. Also these networks model the heterogeneous information like image, text, sound etc. [16].

\subsection{Modeling the Sequence}

These networks are very effective to model the sequence in some tasks such as natural language understanding, speech recognition, machine translation and many more. Recurrent neural networks and convolutional neural networks are very common in these tasks. Convolutional neural networks achieve this great functionality with filter sliding and recurrent neural networks achieve this functionality with its internal memory states. [17].

\subsection{Flexibility in Deep Learning}

These deep neural networks are highly flexible with the invention of different frameworks like Deeplearning4 $\mathrm{j}$, MXnet, Keras, Tensorflow, Theano, and PyTorch. It is also very easy to combine these frameworks to build a powerful hybrid model. In recommender system domain this hybrid model captures all the factors and characteristics. [18]

\section{CONCLUSION AND FUTURE WORK}

In this paper we present an overview of traditional methods and their shortcomings in the recommender system domain. Our main contribution in this work is to provide some recent deep learning methods and take advantages with the help of deep networks for the recommendation to improve the overall accuracy in recommendation. In future our aim is to build a deep hybrid model with the help of neural networks for recommendation and then analyze its result with traditional models.

\section{REFERENCES}

[1] S. Sivapalan, A. Sadeghian, H. Rahnama, and A. M. Madni, "Recommender systems in e-commerce," in World Automation Congress Proceedings, 2014.

[2] M. Ge, F. Ricci, and D. Massimo, "Health-aware food recommender system," RecSys 2015 - Proc. 9th ACM Conf. Recomm. Syst., pp. 333-334, 2015.
[3] G. A. Sielis, A. Tzanavari, and G. A. Papadopoulos, "Recommender Systems Review of Types, Techniques, and Applications," Encycl. Inf. Sci. Technol. Third Ed. no. November, pp. 7260-7270, 2014

[4] F. Ricci, L. Rokach, B. Shapira, P. B. Kantor, and F. Ricci, Recommender Systems Handbook. 2010.

[5] R. Ali, J. Siddiqui, and S. S. Sohail, "http://www.jestr.org/downloads/Volume10Issue4/fullte xt171042017.pdf," J. Eng. Sci. Technol. Rev., vol. 10, no. 4, pp. 132-153, 2017.

[6] B. T. Betru, C. A. Onana, and B. Bernabe, "Deep Learning Methods on Recommender System: A Survey of State-of-the-art," Int. J. Comput. Appl., vol. 162, no. 10, pp. 975-8887, 2017.

[7] S. Edition, Recommender Systems Handbook. 2015.

[8] J. Lu, D. Wu, M. Mao, W. Wang, and G. Zhang, "Recommender system application developments: A survey," Decis. Support Syst., 2015.

[9] M. M. Najafabadi, F. Villanustre, T. M. Khoshgoftaar, N. Seliya, R. Wald, and E. Muharemagic, "Deep learning applications and challenges in big data analytics," J. Big Data, vol. 2, no. 1, pp. 1-21, 2015.

[10] G. Hinton, "Restricted Boltzmann Machines for Collaborative Filtering Ruslan," J. Comput. Inf. Technol., vol. 6, no. 3, pp. 245-254, 1998.

[11] L. Zheng, "A Survey and Critique of Deep Learning on Recommender Systems by,” no. September, 2016.

[12] Q. Li, X. Zheng, and X. Wu, "Neural Collaborative Autoencoder," pp. 1-12, 2017.

[13] B. Hidasi and A. Karatzoglou, "Recurrent Neural Networks with Top-k Gains for Session-based Recommendations," pp. 370-371, 2017.

[14] K. Sheth, "Deep Neural Networks for HDR imaging," pp. 191-198, 2016.

[15] D. Tikk, B. Hidasi, A. Karatzoglou, O. Sar-Shalom, H. Roitman, and B. Shapira, "RecSys'16 Workshop on Deep Learning for Recommender Systems (DLRS)," pp. 415-416, 2016.

[16] S. Sidana, M. Trofimov, O. Horodnitskii, C. Laclau, Y. Maximov, and M.-R. Amini, "Representation Learning and Pairwise Ranking for Implicit Feedback in Recommendation Systems," vol. 14, no. 8, pp. 1-12, 2017.

[17] G. de S. P. Moreira, F. Ferreira, and A. M. da Cunha, "News Session-Based Recommendations using Deep Neural Networks," 2018.

[18] A. Shatnawi, G. Al-Bdour, R. Al-Qurran, and M. AlAyyoub, "A comparative study of open source deep learning frameworks," 2018 9th Int. Conf. Inf. Commun. Syst. ICICS 2018, vol. 2018-January, no. April, pp. 72 77, 2018. 